\title{
Safety Culture is Not Really a Culture! What?!?! So how do we Change it and why do we call it that?!
}

\author{
Allen J* \\ Safety FM, USA \\ Corresponding author: Jay Allen, Safety FM, USA, Email:
}

jayallen@safetyfocusmoment.com

\section{Commentary}

As part of an interesting conversation recently it has come to mind that people get confused on what a culture is. The word is being used as a buzzword in the industry, so people get attached to it and create a silo just for safety and culture not understanding the full context of what is behind safety in an entire industry or specific company. The truth behind the matter is that safety culture should mean and be understood as a collective mindset shift for organizational transformation.

The biological definition for culture is "the growing of microorganisms, tissue cells, or other living matter in a specially prepared nutrient medium where they are able to proliferate readily". We don't want safety to be a microorganism that works in a perfectly prepared environment that is measurable or quantifiable! So how do we make sure we are making an impact and truly changing the environments we are working in so that safety can proliferate outside of a prepared environment? We work on shifting the mindset by initially focusing on shifting the understanding of what safety is and then work on adjusting the narrative of the industry or company. Safety should be a value not a priority.

\section{Steps to Help Create a Mindset Shift}

1. Knowledge is power! People should understand that the most comprehensive fact about safety is that there is not scorecard that can track positive changes. If you think about it in arithmetic terms the only way to say there was a good safety day is to say that 0 incidents occurred. $0+0+0=0$. Since you can't add it or subtract it or multiply it or divide it the limit form of 0 becomes indeterminate.
How do we change something from the top down if all we can go in and say is today we had a zero quarter! It's hard to speak to C-suite or operational executives who live in the language of numbers and ask them to understand that 0 is the winner in safety, but if everyone is educated they will understand and be more open to safety related concepts and viewing safety achievements with a positive mindset. "Not everything that can be counted counts, and not everything that counts can be counted" Albert Einstein

2. Get all levels involved in new initiatives and in developing practices that can be used every day as part of the flow of work to reinforce new mindsets. You can only change the overall flow if you involve everyone from the bottom to top! Once the mindset is reinforced and created it is internalized and closer to becoming a value at an organization instead of a priority. Values are internal, mindsets are internal, and therefore safety becomes an internal mindset. "The aim of education is the knowledge, not of facts, but of values" William S. Burroughs

3. Help individuals and teams create new "now vs. then" stories through continuous learning, improvement, and growth opportunities. Provide an overall goal and empower them to achieve it with the company's best interests for safety at heart. This means don't give steps or instructions! Just ask them to create it and allow operational creativity to bring forward change. From there begin to focus on "now vs then stories" so the history of the transformation is transparent. The 


\section{Ergonomics International Journal}

narrative a department/company tells itself and that the department/company believes can either prevent change from happening or allow new skills to blossom. "Now vs then" stories foster a narrative that believes in change and links their contributions to the overall transformation which then adjusts and internalizes their beliefs and mindset. "Your beliefs become your thoughts, your thoughts become your words, your words become your actions, your actions become your habits, your habits become your values, and your values become your destiny." Mahatma Gandhi
Understanding that safety is part of the core of a business and promoting that concept through education is the only way we can truly affect the transformation of any company. Remember we are not just changing a safety culture but working to adjust a company mindset that needs to be value oriented and internalized.

Happy mindset shifting!

*Thanks provided to Tanmay Vora for another amazing

Sketchnote http://qaspire.com/2015/11/23/mindset -shifts-for-organizational-transformation/* 